\title{
Composition and stability of intestinal microbiota of healthy children within a Dutch population
}

Citation for published version (APA):

de Meij, T. G., Budding, A. E., de Groot, E. F., Jansen, F. M., Kneepkens, C. M., Benninga, M. A., Penders, J., van Bodegraven, A. A., \& Savelkoul, P. H. (2016). Composition and stability of intestinal microbiota of healthy children within a Dutch population. Faseb Journal, 30(4), 1512-1522. https://doi.org/10.1096/fj.15-278622

Document status and date:

Published: 01/04/2016

DOI:

10.1096/fj.15-278622

Document Version:

Publisher's PDF, also known as Version of record

Document license:

Taverne

Please check the document version of this publication:

- A submitted manuscript is the version of the article upon submission and before peer-review. There can be important differences between the submitted version and the official published version of record.

People interested in the research are advised to contact the author for the final version of the publication, or visit the DOI to the publisher's website.

- The final author version and the galley proof are versions of the publication after peer review.

- The final published version features the final layout of the paper including the volume, issue and page numbers.

Link to publication

\footnotetext{
General rights rights.

- You may freely distribute the URL identifying the publication in the public portal. please follow below link for the End User Agreement:

www.umlib.nl/taverne-license

Take down policy

If you believe that this document breaches copyright please contact us at:

repository@maastrichtuniversity.nl

providing details and we will investigate your claim.
}

Copyright and moral rights for the publications made accessible in the public portal are retained by the authors and/or other copyright owners and it is a condition of accessing publications that users recognise and abide by the legal requirements associated with these

- Users may download and print one copy of any publication from the public portal for the purpose of private study or research.

- You may not further distribute the material or use it for any profit-making activity or commercial gain

If the publication is distributed under the terms of Article $25 \mathrm{fa}$ of the Dutch Copyright Act, indicated by the "Taverne" license above, 


\section{Vaccine Discovery and Development: Lessons from COVID-19}

\section{Free eBook}

Emerging infectious diseases (EIDs) can evolve into a global healthcare crisis or pandemic. Scientists have previously required years to develop vaccines or therapeutics. The use of high throughput technology can greatly broaden the insights collected during discovery, augment efficiency and safety of handling EIDs, and shorten timelines.

Download this publication for an overview of many lessons learned in virology, immunology, and vaccine research during COVID-19 vaccine development.

\section{Download here}




\title{
Composition and stability of intestinal microbiota of healthy children within a Dutch population
}

\author{
Tim G. J. de Meij, ${ }^{* 1,2}$ Andries E. Budding, ${ }^{\dagger, 1}$ Evelien F. J. de Groot,* Fenna M. Jansen,* \\ C. M. Frank Kneepkens, ${ }^{*}$ Marc A. Benninga ${ }^{\ddagger}{ }^{\ddagger}$ John Penders, ${ }^{\S}$ Adriaan A. van Bodegraven, ${ }^{\top,}, \|$ \\ and Paul H. M. Savelkoul ${ }^{\dagger, \S}$ \\ *Department of Pediatric Gastroenterology, ${ }^{\dagger}$ Department of Medical Microbiology and Infection \\ Control, and "Department of Gastroenterology, VU University Medical Center, Amsterdam, The \\ Netherlands; ${ }^{\ddagger}$ Department of Pediatric Gastroenterology, Academic Medical Center, Amsterdam, The \\ Netherlands; ' Department of Medical Microbiology, Maastricht University Medical Center, Maastricht, \\ The Netherlands; and ${ }^{\top}$ Department of Gastroentorology, Internal Medicine, Intensive Care Medicine \\ and Geriatrics Zuyderland Medical Center, Heerlen-Sittard-Geleen, The Netherlands
}

\begin{abstract}
Numerous diseases linked to microbial imbalance can be traced back to childhood, illustrating the impact of the juvenile microbiota development from infancy toward adulthood. However, knowledge on this subject is currently very limited. The primary aim of this study was to characterize composition and short- and long-term stability of the intestinal microbiota in healthy children. Between November 2011 and June 2014, 61 children 2 to 18 yr of age from different areas in The Netherlands were included and instructed to collect fecal samples weekly, for $6 \mathrm{wk}$, and a followup sample after $18 \mathrm{mo}$. The intergenic spacer profiling technique (IS-pro) was used to analyze all available fecal samples. Microbial diversity was calculated by the Shannon diversity index and individual compositional stability by comparing all collection time points. Microbial stability varied per phylum $(P<0.0005)$, declined rapidly in a short time period, and subsequently stabilized on the long run with very gradual variation, leading to an overall compositional stability of $70 \%$ on average over a period of 18 mo. Higher species diversity was correlated to a higher overall compositional stability $(P<0.001)$. We observed an age-independent bacterial shared core consisting of a limited number of species. In conclusion, in this study, we showed that microbial composition stability in children varied per phylum, at both short-term and long-term intervals. Healthy children seem to share a microbiome core consisting of a limited number of species.-De Meij, T. G. J., Budding, A. E., de Groot, E. F. J., Jansen, F. M., Kneepkens, C. M. F., Benninga, M. A., Penders, J., van Bodegraven, A. A., Savelkoul, P. H. M. Composition and stability of intestinal microbiota of healthy children within a Dutch population. FASEBJ. 30, 1512-1522 (2016). www.fasebj.org
\end{abstract}

Key Words: core $\cdot 454$-pyrosequencing $\cdot$ IS-pro $\cdot$ diversity $\cdot$ microbiome

Abbreviations: FAFV, Firmicutes, Actinobacteria, Fusobacteria, and Verrucomicrobia; IS, intergenic spacer; OTU, operational taxonomic unit
The human body harbors trillions of microbes belonging to hundreds of different species, of which the vast majority reside in the gastrointestinal tract. Investigation into the pathophysiologic significance of our microbial symbionts has only recently been boosted by new DNA-based detection methods. It has become clear that the intestinal microbiota plays a crucial role in maintaining intestinal and overall health, including nutrient digestion and regulation of host metabolism and immune system (1-4). Disruption of the homeostasis between the gut microbiota and host has been linked to gastrointestinal and systemic diseases, such as inflammatory bowel disease, necrotizing enterocolitis, atopy, autoimmune diseases, and obesity (5-9). These observations have reinforced the potential of microbiota characterization as a diagnostic biomarker for health and disease, opening avenues toward development of novel, microbiota-targeted preventative and therapeutic strategies. For recognition of disease-specific microbial patterns and assessment of their role in (the course of) disease, detailed understanding of the intestinal microbiota in terms of composition and physiologic temporal variations is essential (10). Current knowledge on composition and dynamics of the human gut microbiota is limited, methodologically inconsistent, and largely based on studies in adults, describing a fairly stable composition over time. Studies on composition and temporal stability in children have almost exclusively focused on the first years of life, a period characterized by a highly dynamic and shifting intraindividual bacterial composition (11-13). Information on the development and stability of the microbiota from infancy toward adulthood is yet very limited. The fact that numerous

\footnotetext{
${ }^{1}$ These authors contributed equally to this work.

${ }^{2}$ Correspondence: Department of Pediatrics, VU University Medical Center, De Boelelaan 1117, 1081 HV, Amsterdam, The Netherlands. E-mail: t.demeij@vumc.nl

doi: 10.1096/fj.15-278622

This article includes supplemental data. Please visit http:// www.fasebj.org to obtain this information.
} 
diseases linked to microbial imbalance can be traced back to this specific period illustrates the importance and impact of the juvenile microbiota (14-16). Characterization of the normal microbiota in children and its development from infancy toward adulthood is central to enlarge knowledge about microbiota-related pathology.

The primary aim of this study was to characterize composition and short-term and long-term stability of the intestinal microbiota in healthy children from 2 to $18 \mathrm{yr}$ of age.

\section{MATERIALS AND METHODS}

\section{Subjects and study design}

A heterogeneous cohort of healthy Dutch children was included to represent a cross section of children from all ages. For this purpose, we approached parents of children in the age range of 2-18yr, visiting primary and secondary schools in both urban and rural areas in 5 different provinces of the Netherlands (NoordHolland, Zuid-Holland, Overijssel, Friesland, and Flevoland), and invited them to participate. To focus exclusively on temporal gut microbiota dynamics during state of health, strict criteria for exclusion were applied: use of antibiotics or immune modulating agents within 6 mo prior to the study, culture-proven infectious gastroenteritis in the last 6 mo prior to inclusion, history of surgery of the gastrointestinal tract (except appendectomy), or a diagnosis of chronic gastrointestinal disease, such as celiac disease, functional constipation, short bowel syndrome, or inflammatory bowel disease. Subjects (or their parents) completed a questionnaire on the following items: age, length and weight, pregnancy duration, mode of delivery, type of neonatal feeding (breastfeeding or formula feeding), use of antibiotics in the first year of life, current medication, and defecation pattern.

This study was approved by the Medical Ethics Committee of VU University Medical Center and was performed in accordance with the ethical standards laid down in the 1964 Declaration of Helsinki and its later amendments.

\section{Sample size calculation}

A formal power analysis could not be performed for this study because no previous data on microbiota stability in healthy children were available.

\section{Sampling}

Sterile plastic containers and an information letter were provided to parents and children, with instructions on procedure of collection and storage of the fecal samples. Children (or their parents) were instructed to collect fecal samples (approximately $2 \mathrm{~g}$ ) for $6 \mathrm{wk}$ at weekly sampling intervals and a follow-up sample after $18 \mathrm{mo}$. Fresh fecal samples were immediately stored in the freezer at home at $-20^{\circ} \mathrm{C}$. For collection of the 18-mo follow-up samples, parents were contacted by telephone or e-mail. Nonresponders received a maximum of 2 reminders. At time of collection of the follow-up fecal sample, information was obtained on use of antibiotics and major changes in health status during the last $18 \mathrm{mo}$, such as a diagnosis of (chronic) gastrointestinal disease. DNA extraction was performed after collection of those frozen fecal samples collected at weekly intervals, and DNA extraction of the follow-up samples was performed within 3 mo following collection.

\section{DNA extraction and sample preparation}

DNA was extracted from fecal samples with the easyMag extraction kit according to the manufacturer's instructions (Biomérieux, Marcy l'Etoile, France). Feces (100-400 mg) was placed in an Eppendorf tube with $200 \mu$ l of nucliSens lysis buffer and vortexed. Tubes were incubated while shaking for $5 \mathrm{~min}$ at room temperature. After centrifugation (13,000 rpm; $2 \mathrm{~min}), 100 \mu l$ supernatant was transferred to an easyMAG isolation container containing $2 \mathrm{ml}$ nucliSENS lysis buffer. This suspension was incubated for $10 \mathrm{~min}$ at room temperature, after which $70 \mu \mathrm{l}$ of magnetic silica beads were added. The easyMAG automated DNA isolation machine was used following the "specific A" protocol, eluting DNA in $110 \mu \mathrm{l}$ buffer. All fecal samples were analyzed by intergenic spacer profiling (IS-pro), and the first samples collected (baseline samples) were also analyzed by 454-pyrosequencing (17).

IS-pro

For IS-pro, DNA samples were diluted 1:10. Amplification of IS regions was performed with the IS-pro assay (IS-Diagnostics, Amsterdam, The Netherlands) according to the protocol provided by the manufacturer. IS-pro differentiates bacterial species by the length of the 16S-23S rDNA IS region with taxonomic classification by phylum-specific fluorescently labeled PCR primers (17). The procedure consists of 2 PCR reactions, together covering the phyla Firmicutes, Actinobacteria, Fusobacteria, Verrucomicrobia, Bacteroidetes, and Proteobacteria. The first PCR reaction contains 2 different fluorescently labeled forward primers and 3 unlabeled reverse primers. The first forward primer is specific for the phyla Firmicutes, Actinobacteria, Fusobacteria, and Verrucomicrobia (FAFV), and the other labeled forward primer is specific for the phylum Bacteroidetes. The second PCR contains a forward-labeled primer specific for the phylum Proteobacteria and a combination of 7 reverse primers, together covering the phylum Proteobacteria.

The IS-pro primers have been extensively evaluated for coverage of the various phyla included in the assay. An in silico analysis was performed on all 16S-23S sequences available at GenBank in April 2014 (8990 sequences). Here, matches for both forward and reverse primers were found in $95 \%$ of Firmicutes (3129/3303), 99\% of Actinobacteria (814/815), 88\% Bacteroidetes $(245 / 280)$, and $98 \%$ of Proteobacteria sequences (3714/3803). Furthermore, to confirm in silico results and to evaluate reproducibility of IS profiles within species, IS-pro reactions were performed on 588 cultured bacterial strains, belonging to 189 species from the phyla covered by the IS-pro primers. These findings confirmed in silico predictions. The data from the in silico and in vitro analyses were combined to form a database used in translation of profiles to species names.

Amplifications were carried out on a GeneAmp PCR system9700 (Applied Biosystems, Foster City, CA, USA). After PCR, $5 \mu$ l PCR product was mixed with $20 \mu$ formamide and $0.2 \mu \mathrm{l}$ custom size marker (IS-Diagnostics). DNA fragment analysis was performed on an ABI Prism 3500 Genetic Analyzer (Applied Biosystems).

\section{4-Pyrosequencing}

Amplicon libraries for pyrosequencing of the 16S rDNA V1-V3 regions were generated using 2 primers: the first primer was a barcoded reverse primer consisting of the 454 Titanium platform A linker sequence (5'-CCATCCCTGCGTGTCTCCGACTCAG$3^{\prime}$ ), a key (barcode of 6-8 nt as described by the HMP consortium

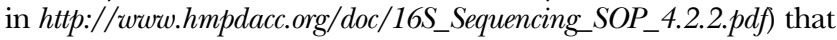
was unique for each sample, and the 16S rRNA 534R primer sequence 5 '-ATTACCGCGGCTGCTGG-3'. The second primer was a forward primer consisting of a 9:1 mixture of 2 oligonucleotides, 5' -B-AGAGTTTGATCMTGGCTCAG-3' and 5'-BAGGGTTCGATTCTGGCTCAG- $3^{\prime}$, where B represents the B linker (5'-CCTATCCCCTGTGTGCCTTGGCAGTCTCAG-3') followed by the $16 \mathrm{~S}$ rRNA $8 \mathrm{~F}$ and 8 F-Bif primers, respectively (18). 
PCR amplifications (in a volume of $50 \mu \mathrm{L}$ ) were performed using $1 \times$ FastStart High Fidelity Reaction Buffer, $1.8 \mathrm{mM} \mathrm{MgCl}_{2}$, $1 \mathrm{mM}$ dNTP solution, 5 U FastStart High Fidelity Blend Polymerase (from the High Fidelity PCR System; Roche, Indianapolis, IN, USA), $0.2 \mu \mathrm{M}$ reverse primer, $0.2 \mu \mathrm{M}$ of the barcoded forward primer (unique for each sample), and $1 \mu \mathrm{L}$ of template DNA. PCR cycle conditions were as follows: an initial denaturation at $94^{\circ} \mathrm{C}$ for $3 \mathrm{~min}$, followed by 25 cycles of denaturation at $94^{\circ} \mathrm{C}$ for $30 \mathrm{~s}$, annealing at $51^{\circ} \mathrm{C}$ for $45 \mathrm{~s}$, and extension at $72^{\circ} \mathrm{C}$ for $5 \mathrm{~min}$, and a final elongation step at $72^{\circ} \mathrm{C}$ for $10 \mathrm{~min}$. Amplicons $(20 \mu \mathrm{L})$ were purified using AMPure XP purification (Beckman Coulter, Beverly, MA, USA) according to the manufacturer's instructions and eluted in $25 \mu \mathrm{l} \mathrm{TE}$ buffer $1 \times$ solution, $\mathrm{pH}$ 8.0, and low EDTA.

Amplicon concentrations were determined by the Quant-iT PicoGreen dsDNA reagent kit (Thermo Fisher Scientific, Grand Island NY, USA) using a Victor3 Multilabel Counter (Perkin Elmer, Waltham, MA, USA). Amplicons were mixed in equimolar concentrations. 454-Sequencing was performed on a GS FLX Titanium PicoTiterPlate with a GS FLX pyrosequencing system (Roche, Branford, CT, USA).

\section{Data analysis}

\section{IS-Pro}

Preprocessing was carried out with the IS-Pro proprietary software suite (IS-Diagnostics) and resulted in microbial profiles. Three levels of information were obtained: color of peaks sorts species into the phyla FAFV, Bacteroidetes, and Proteobacteria, which are the main phyla present in the human gastrointestinal tract. Length of the 16S-23S rDNA IS region, displayed by number of nucleotides, can subsequently be used to identify bacteria at the species level. Specific peak height, measured in relative fluorescence units, reflect the quantity of PCR product. To further analyze the obtained data, each peak in a profile was considered as an operational taxonomic unit (OTU) and its corresponding intensity as its abundance. Species determination of IS-pro peaks was done by matching of profiles to a database of IS profiles of known bacterial species. In previous analyses, the effect of sampling on IS profiles was assessed. The correlation of profiles from samples of the same excrement was found to be $96 \%$ for Bacteroidetes and $90 \%$ for FAFV (17).

\section{4-Pyrosequencing}

Raw pyrosequencing reads were passed through quality filters to reduce the overall error rate using Mothur version 1.23 (19). Only sequences with perfect proximal primer fidelity and a threshold quality score of $\geq 20$, a read length between 200 and 540 nucleotides, a maximum of 1 ambiguous base call, and a maximum homopolymer length of 6 were retained for further analysis. Data processing was done with Quantitative Insights Into Microbial Ecology, version 1.5.1 (20). Barcodes were used to identify sequences from each individual sample. The UCLUST algorithm was used to cluster sequences into OTUs or phylotypes based on $97 \%$ similarity (species level) against the Greengenes reference set (21). The following nondefault search parameters for Uclust algorithm were applied: maxrejects $=100$ and stepwords $=16$. Creation of new clusters for sequences that did not cluster to reference sequences within the given similarity threshold was disabled to further reduce the influence of pyrosequencing errors. Finally, OTUs that were only recovered in a single sample were removed from the dataset. In total, 1,403,456 sequences were recovered from 454 sequencing. After trimming, filtering, binning, and clustering, 722,109 sequences (mean $\pm \mathrm{SD}$ length: $326 \pm 76.1$ bases), ranging from 6195 to 18,662 sequences per sample, remained for further analysis. For downstream analyses, data were rarefied at a sequencing depth of 6195 sequences per sample.

\section{Diversity and stability analysis}

Diversity and stability analysis was performed on the IS-pro data. Microbial diversity was calculated as the Shannon diversity index based on the resulting profiles by conventional statistics. Diversity was calculated both per phylum and for overall microbial composition (by pooling the phyla FAFV, Bacteroidetes, and Proteobacteria together). Diversity analysis was performed with the R 2.15.2 software package. Data visualizations and fitted curves were done with the Spotfire software package (Tibco, Palo Alto, CA, USA).

In this study, compositional stability is defined as intraindividual resistance to change in relative abundances of species over time, quantified by cosine distance (lower distance value represents higher stability), and expressed as a percentage value (e.g., when 2 fecal samples of 1 individual collected over time would have identical microbial composition, compositional stability is $100 \%$ ). The compositional stability of fecal microbiota of children through time was estimated by comparing all intervals per individual (i.e., for 1 week stability, all 1-wk intervals were compared; for 2-wk stability, all 2-wk intervals were compared and so on). For 18 mo stability, the follow-up sample was compared with all other samples to minimize impact of potential outliers on this analysis. Sample compositions were compared by calculating cosine distances for log2-transformed data per phylum and for the phyla FAFV, Bacteroidetes, and Proteobacteria combined (17). Multivariate ANOVA was performed, when appropriate using a Greenhouse-Geisser correction, taking time and phylum as within-subject variables. Dissimilarities between samples, or between-sample diversity, were represented in a dissimilarity matrix that was built using the cosine distance measure. Given 2 vectors of attributes ( 2 profiles in our case), A and $\mathrm{B}$, the cosine dissimilarity is represented using a dot product and magnitude as

$$
\text { Dissimilarity }=1-\cos (\theta)=1-\frac{\sum_{i=1}^{n} A_{i} \times B_{i}}{\sqrt{\sum_{i=1}^{n}\left(A_{i}\right)^{2}} \times \sqrt{\sum_{i=1}^{n}\left(B_{i}\right)^{2}}}
$$

\section{Network analysis}

To analyze the potential presence of a microbial core at the level of OTU, a concept defined as a fixed set of bacterial species present in more than $80 \%$ of all healthy children, a network visualization approach was taken. An anchored bipartite network was generated from all IS-pro data (22). Samples were represented as anchored nodes in a circular layout and bacterial OTUs as unanchored nodes for which the position was calculated as the center of mass of all connected sample nodes. Edges were created between a sample and an OTU node when that particular OTU was present in that particular sample. OTUs that were present in only a single sample were placed outside the circle of anchored sample nodes. The network visualization was made in Cytoscape 3.1.0 (23). For this particular visualization, a custom plugin was made. The color of nodes was determined by degree and the size by betweenness centrality (24). Finally, edges were bundled with the "bundle edges" plugin. Parameters were as follows: number of handles, 3; Spring constant, 0.003; compatibility threshold, 0.3; maximum iterations. For an illustrated step-by-step description of how the network was built, refer to Supplemental Data.

\section{RESULTS}

\section{Participants}

Between November 2011 and June 2014, 61 children were included in this study; each child collected 1 fecal sample 
per wk for 6 wk. Forty-five children also collected a followup sample after $18 \mathrm{mo}$. None of the children and parents reported relevant changes in health state between collection of first samples and follow-up sample; 2 children used antibiotics for a respiratory tract infection. All children reported normal defecation patterns with more than 3 bowel movements per wk. All parents were born in Western Europe, except for 3 couples with diverse backgrounds (South Korea, Aruba, and Peru). The characteristics of the participants are summarized in Table 1.

\section{Diversity and stability}

Microbial compositional stability through time varied per time point $(P<0.0005)$ and per phylum $(P<$ $0.0005)$, with Bacteroidetes showing the highest compositional stability, followed by Proteobacteria and FAFV. Stability declined quite rapidly for short intervals, but then stabilized at a level that declined further only very gradually. $P$ values for longitudinal decrease were $3.41 \times 10^{-27}$ for Proteobacteria, $5.32 \times 10^{-19}$ for Firmicutes, and $1.33 \times 10^{-14}$ for Bacteroidetes. Overall compositional stability was on average $70 \%$ over a period of $18 \mathrm{mo}$ (Fig. 1). Also at the level of the individual, it was found that Bacteroidetes composition was most stable, whereas the compositional stability of FAFV and Proteobacteria was lower on average. Finally, there seemed to be a slight increase in compositional stability with age, but this effect was not statistically significant (Fig. 2A). The identified variations in microbiota

TABLE 1. Subject characteristics

\begin{tabular}{|c|c|}
\hline Characteristic & Value \\
\hline Number of subjects $(n)$ & 61 \\
\hline Age [median (IQR)] (yr) & $7.8(6.7)$ \\
\hline Range (yr) & $2.1-17.8$ \\
\hline Male $(\%)$ & 48 \\
\hline 18-mo follow-up sample $(n)$ & 45 \\
\hline BMI [median (IQR)] & $15.9(3.6)$ \\
\hline \multicolumn{2}{|l|}{ Area inclusion ${ }^{a}(n)$} \\
\hline Agriculture & 8 \\
\hline Urban & 53 \\
\hline \multicolumn{2}{|l|}{ Mode of delivery $(n)$} \\
\hline Vaginal & 53 \\
\hline Caesarian delivery & 8 \\
\hline \multicolumn{2}{|l|}{ Pregnancy duration $(n)$} \\
\hline$<37$ wk & 3 \\
\hline $37-42$ wk & 58 \\
\hline \multicolumn{2}{|l|}{ Neonatal feeding $(n)$} \\
\hline Exclusively formula fed & 1 \\
\hline Breastfeeding & 60 \\
\hline \multicolumn{2}{|l|}{ Duration of breastfeeding $(n)$} \\
\hline$<3 \mathrm{mo}$ & 10 \\
\hline $3-6 \mathrm{mo}$ & 26 \\
\hline$>6 \mathrm{mo}$ & 24 \\
\hline Antibiotic use first year of life $(n)$ & 13 \\
\hline \multicolumn{2}{|l|}{ Medication at time of sampling $(n)$} \\
\hline Probiotics & 6 \\
\hline Birth control pills & 1 \\
\hline Salbutamol inhalator & 2 \\
\hline
\end{tabular}

BMI, body mass index; IQR, interquartile range. ${ }^{a}$ Urban area includes towns and cities; children from agriculture origin live on a farm.

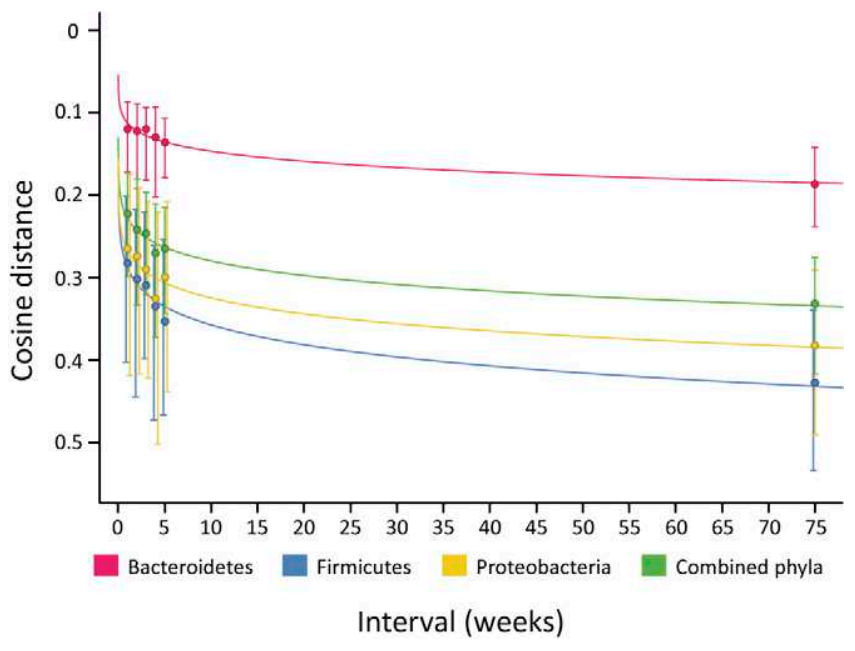

Figure 1. Compositional stability of fecal microbiota of children through time. Dots represent median cosine distance of microbiota composition ( $y$ axis) per time interval ( $x$ axis). Microbiota stability declined quite rapidly for short intervals, but then stabilized at a level that declined further only very gradually, with the highest stability for phylum Bacteroidetes (red), followed by Protebacteria (yellow), and FAFV (blue). Overall compositional stability is depicted in green.

composition through time far exceeded the expected variation induced by multiple testing (i.e., taking a sample from the same source twice and performing the whole analysis procedure twice, from DNA isolation to final data analysis), indicating that the measured effect was indeed temporal variation.

Diversity of fecal samples was highest for the phylum Bacteroidetes with a median Shannon diversity index of 2.81, followed by the phyla Proteobacteria (2.62) and FAFV (2.46) at baseline. Stability of diversity indices over time was also assessed and was found to be highest for the phylum Bacteroidetes (Fig. 3). Diversity index for the phyla FAFV and Proteobacteria appeared to be more variable over time. A further assessment was done to analyze microbial diversity, its stability, and relation to age on an individual level. The results are shown in Fig. $2 B$. Here, diversity was highest and most stable over time for the phylum Bacteroidetes. For the phyla FAFV and Proteobacteria, median diversity indices and individual variation were much larger than for the phylum Bacteroidetes.

Importantly, compositional stability and stability of diversity of the measured phyla FAFV, Bacteroidetes, and Proteobacteria were highly individual specific. A higher diversity was correlated with higher stability for these phyla separately and for the combined microbiota. Also, higher diversity was correlated to a higher stability of the diversity index (Fig. 4). Children exposed to antibiotics in their first year of life had similar Shannon diversity indices compared with unexposed children (data not shown).

When allocating children to 1 of 3 defined age groups (blocks of $5 \mathrm{yr}$ : from 2 to $7 \mathrm{yr}$ of age; from 8 to $13 \mathrm{yr}$; and from 13 to $18 \mathrm{yr}$ ), no statistically significant differences were observed in composition, stability, and diversity between these 3 age cohorts with an unpaired Student's $t$ test. Furthermore, no particular clustering was seen based on sex. 


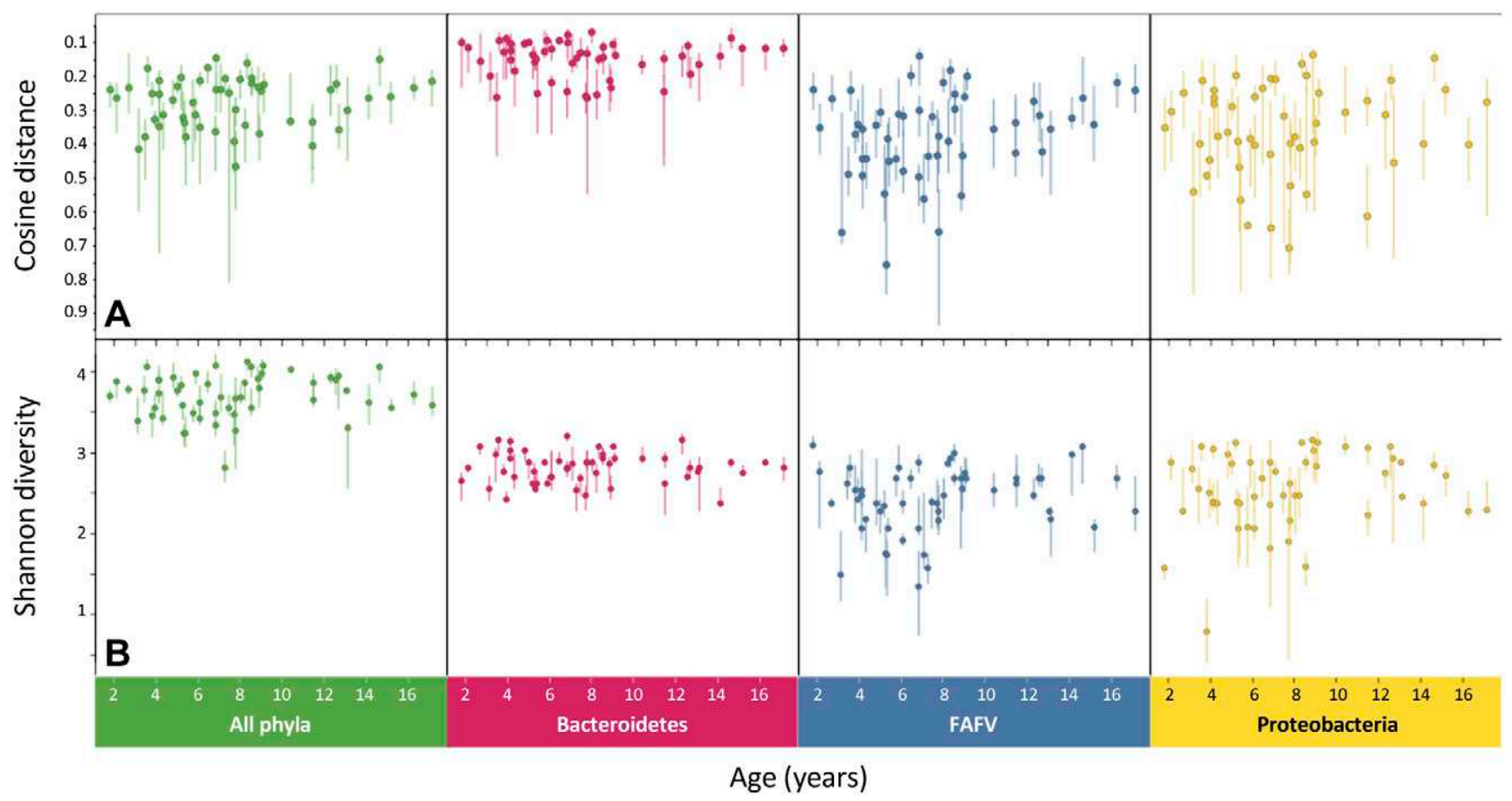

Figure 2. Individualized analyses on IS-pro data for the phyla Bacteroidetes (red), FAFV (blue), Proteobacteria (yellow), and all phyla (green), sorted by age (in years, $x$ axis). Dots represent median values of all samples from a given child, except the 18 mo follow-up sample, plotted as function of age in years. Error bars represent interquartile range (IQR). A) Compositional stability per child sorted by age as expressed by cosine distance between sequential samples (lower values represent higher stability). Compositional stability was highest for Bacteroidetes, whereas the stability for the other phyla appeared more individualized: especially in younger children, interindividual variation in stability of FAFV and Proteobacteria was high. $B$ ) Diversity was highest and most stable for the phylum Bacteroidetes. For the phyla FAFV and Proteobacteria, diversity indices had a larger distribution and were less stable.

\section{Microbiota core}

In the network analysis, a bacterial core consisting of species that were present in the fecal samples of the majority of children became apparent (Fig. 5). This core was dominated by species from the phylum Bacteroidetes, with the genera Bacteroides and Alistipes having the highest average abundances. From the phylum Firmicutes, the families Ruminococcacaea and Lachnospiraceae were

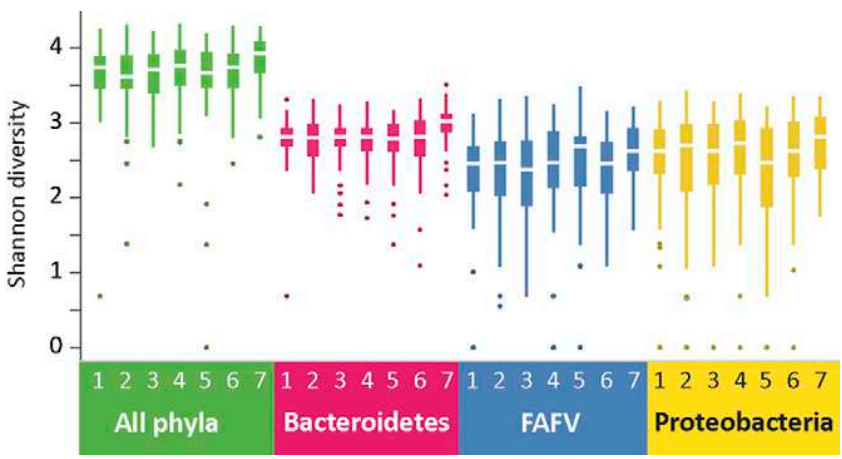

Figure 3. Boxplot of Shannon diversity indices at different time points (T1-6 correspond with sampling number in weeks, T7 is follow-up at 18 months). Bacteroidetes had the highest and most stable diversity at all time points, followed by Proteobacteria and FAFV. Distribution of diversity was considerably larger for the latter phyla than for Bacteroidetes. Notably, at T7, Bacteroidetes diversity seemed to be increased for all children. most dominant. The presence and identity of this shared core was confirmed in all baseline samples with 454pyrosequencing (Fig. 6). Table 2 summarizes the most abundant OTUs in 454 data (present in $>70 \%$ of all subjects) and their matching to the most common species as found with IS-pro. Figure 7 represents IS-pro and 454-sequencing data of the most abundant taxa at the family level, including their relative abundance.

\section{DISCUSSION}

In this study, we showed that microbial composition stability in children is individualized and varied per phylum at both short-term and long-term intervals. This was consistent throughout all age cohorts of youth. Diversity and stability for all bacterial phyla were correlated: diversity of the phylum Bacteroidetes was highest, and this phylum also showed the highest stability compared with the phyla Proteobacteria and FAFV. Finally, we observed the presence of a shared microbial core, consisting of a limited number of species that was present in the majority of healthy children.

\section{Dynamics}

Human gut microbiota stability patterns were first described in 1998 by Zoetendal et al. (25). They showed that the composition of the gut microbiota of 2 adults remained fairly stable over a course of months. Subsequent studies 

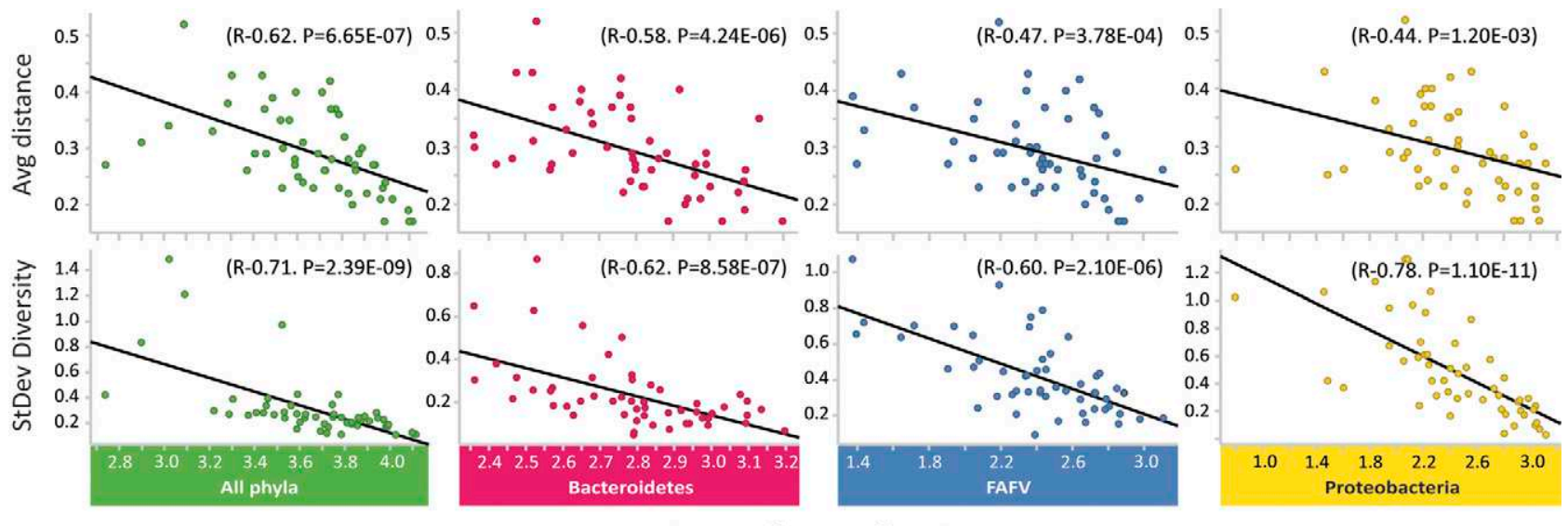

Average Shannon diversity

Figure 4. Correlation of average diversity values per child with average cosine distances (upper) and standard deviation of Shannon diversity index (lower) for the phyla FAFV, Bacteroidetes, and Proteobacteria together (green) and Bacteroidetes (red), FAFV (blue), and Proteobacteria (yellow) seperately. A significant negative correlation was apparent in both analyses for all measured phyla. Children with a higher diversity of their microbiota thus showed a lower variation of that diversity through time, as measured by the standard deviation of diversity, Also, samples with higher diversity are compositionally more similar throughout time, as measured by the average cosine distance between all time points. Spearman rank correlation coefficients and associated $P$ values are depicted for each correlation.

confirmed the existence of relatively stable, hostdependent microbiota, characterized by prominent fluctuations around the average, at both species and phylum levels (26-34). However, this generally stable

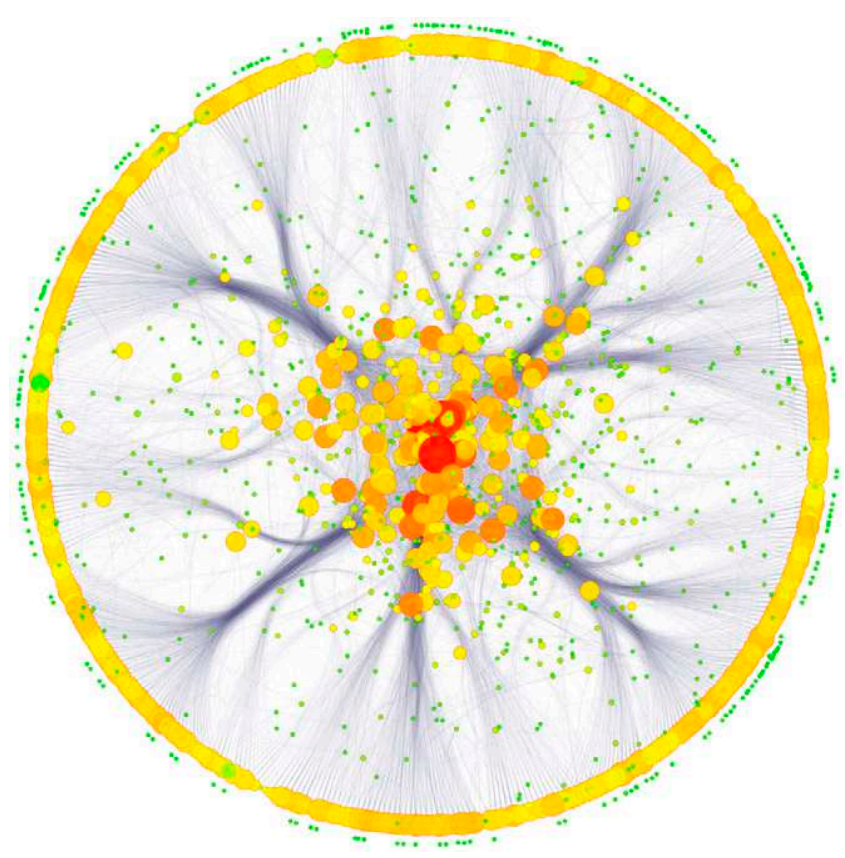

Figure 5. Anchored bipartite network depicting samples from all children at all time points in a circular layout, based on all IS-pro data. All samples of all children are displayed as nodes on the circumference of the circle. Bacterial OTU nodes were placed at the center of gravity of the sample nodes in which the OTUs occur. Thus, the more children who shared a certain OTU, the more central that OTU has been placed. Bacteria that were common in many individuals appeared as a visual core. Size and color of nodes were determined by the number of nodes these were connected to (red, highest connectivity; green, lowest connectivity). Core species appeared in the center, were larger, and had a hue toward red. course has been shown to be affected by lifestyle and major events in human life, such as dietary alterations, enteric infections, and traveling from the developed to the developing world, which could rapidly and profoundly impact microbiota dynamics (35). More recently, 2 studies including 5 and 37 healthy adults, respectively, showed that stability of the gut microbiota differed per phylum $(36,37)$. In adults, the composition of Bacteroidetes and Actinobacteria was more stable and that of Proteobacteria was less stable over time compared with overall stability. Firmicutes showed a more variable pattern, consisting of both highly stable and less stable members. In our study including 61 children, we also observed that stability varied per phylum, with the phylum Bacteroidetes as the most stable component of the gut microbiota, followed by the Proteobacteria and FAFV. Similar to the observations in adults by others, we noticed that microbiota stability in children between 2 and $18 \mathrm{yr}$ of age declined quite rapidly for short intervals, but then stabilized at a level that declined further only very gradually. In adults, on average, $70 \%$ and $60 \%$ of identified strains were preserved over the course of 1 and $5 \mathrm{yr}$, respectively. We found an overall compositional stability of on average $70 \%$ over a period of $18 \mathrm{mo}$.

Phylum-specific temporal shifts in composition may be due to variation in individual diets, because most prominent fluctuations have been described in bacterial species involved in food digestion, such as Bifidobacterium adolescentis and Parabacteroides distasonis, and possessing carbohydrate-degrading enzymes, such as Clostridium clostridioforme and Faecalibacterium prausnitzii (38). This concept of dietary effects on intraindividual microbial dynamics was supported by a study on temporal compositional stability that compared Bangladeshi and U.S. children (39).

Remarkably, we noticed an increase in diversity index of the phylum Bacteroidetes at 18 mo of follow-up, whereas no increase was observed for the other phyla. In this 


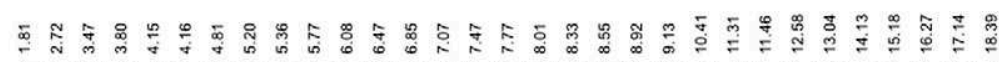

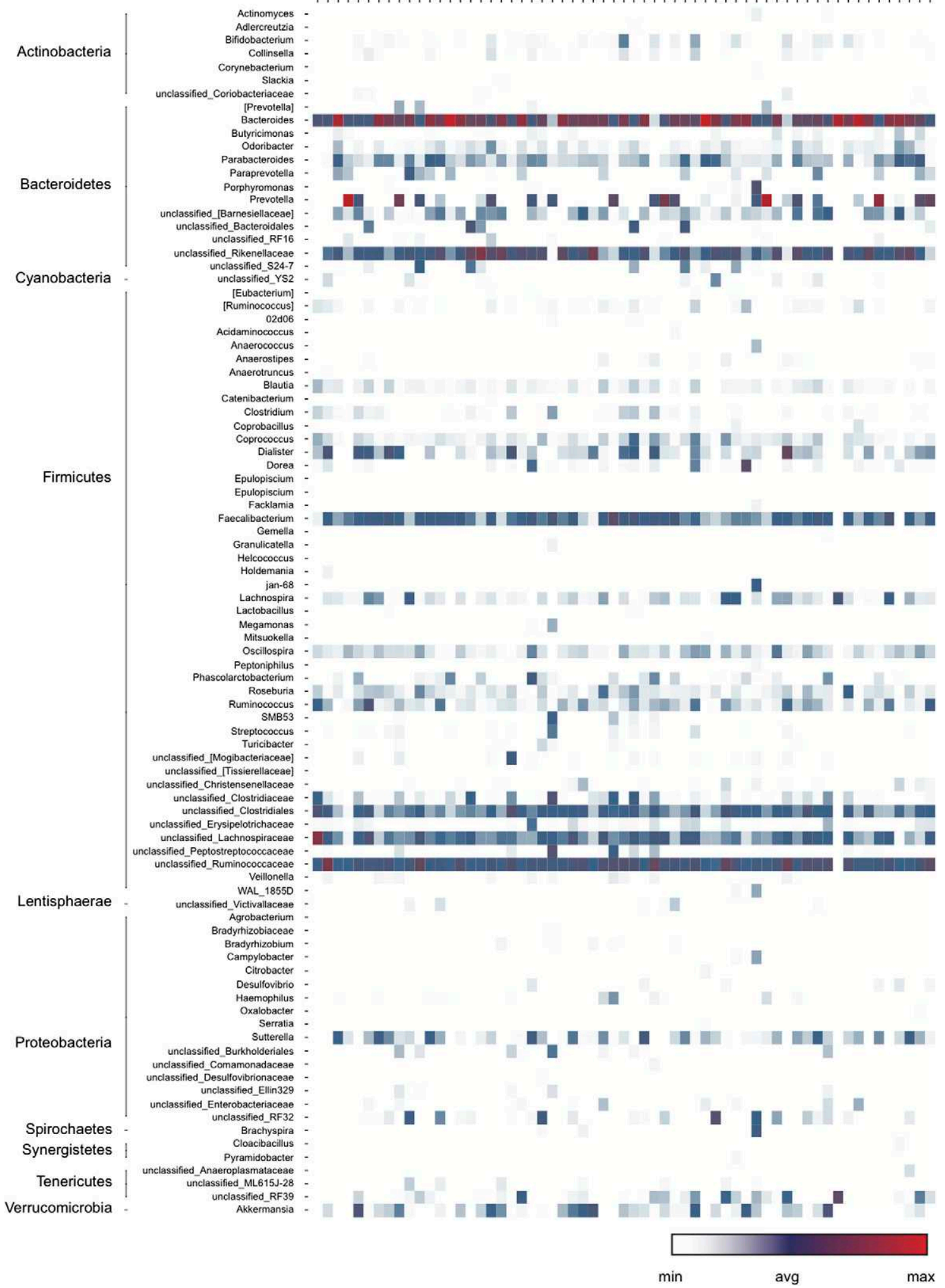

Figure 6. Heatmap of 454-pyrosequencing data of all baseline samples, sorted by age (on $x$ axis), with different species on $y$ axis. Only taxa with an abundance $>0.1 \%$ are displayed. Intensity of colors reflects relative abundance in each sample. It can be clearly seen here that some genera occur in almost all individuals (shown as horizontal lines). These genera correspond with the core species as detected by IS-pro data. 
TABLE 2. Matching of 454-sequencing and IS-pro data for identification of core species

\begin{tabular}{lcc}
\hline \hline Core species & 454-Sequencing [prevalence (\%)] & IS-pro peaks \\
\hline Alistipes finegoldii & $60 / 61(98)$ & $231 / 396 / 400 / 406$ \\
Faecalibacterium prausnitzii & $60 / 61(98)$ & $313 / 318 / 320$ \\
Bacteroides vulgatus & $59 / 61(97)$ & 479 \\
Alistipes putredinis & $58 / 61(95)$ & 235 \\
Bacteroides fragilis & $58 / 61(95)$ & 537 \\
Prevotella spp. & $58 / 61(95)$ & 437 \\
Odoribacter splanchnicus & $56 / 61(92)$ & 308 \\
Parabacteroides distasonis & $54 / 61(89)$ & 464 \\
Sutterella wadsworthensis & $53 / 61(87)$ & $660-663 / 871$ \\
Unclassified Ruminococcaceae & $53 / 61(87)$ & 888 \\
Unclassified Firmicute & $51 / 61(84)$ & 257 \\
Sutterella spp. & $50 / 61(82)$ & 651 \\
Unclassified Proteobacteria & $48 / 61(79)$ & 941 \\
Unclassified Firmicute & $48 / 61(79)$ & 361 \\
Escherichia coli & $47 / 61(77)$ & $735 / 828$ \\
Bacteroides spp. & $46 / 61(75)$ & 474 \\
Bacteroides spp. & $45 / 61(74)$ & 548 \\
Unclassified Firmicute & $45 / 61(74)$ & 541 \\
Akkermansia muciniphila & $43 / 61(70)$ & $598 / 602$ \\
Unclassified Firmicute & $43 / 61(70)$ & 558 \\
Unclassified Proteobacteria & $43 / 61(70)$ & 747 \\
\hline
\end{tabular}

The most abundant OTUs as detected by 454-pyrosequencing data and their matching to the most common species as found with IS-pro are displayed, including a column displaying prevalence of each taxon.

respect, it should be noted that the majority of 18 mo follow-up samples were collected during the summer, whereas the first weekly samples were collected mainly during the winter. Seasonal shifts in microbiota composition, including shifts in Bacteroidetes, have recently been described in adults, and have been attributed to seasonal dietary differences $(40)$.

We also observed a positive correlation between diversity and stability. This finding is of general ecological interest as this has been a long debated issue for nonmicrobial ecosystems (41). Furthermore, this finding may also be of particular interest for the role of microbiota in health and disease, because low microbial diversity has been linked to various diseased states (42). It is interesting to observe that stability of diversity may be higher than compositional stability, as we found for the phylum Proteobacteria. This suggests succession of different species within the population while maintaining the same level of diversity.

\section{Composition and core}

Until recently, it has been assumed that at the age of about $3 \mathrm{yr}$, the gut microbiota composition has converged toward a relatively stable, adult-like pattern (11, $43,44)$. In 2 recent studies, however, substantial compositional differences between children of different ages and adults were described, suggesting that, also from a microbial point of view, children cannot be regarded as miniaturized adults $(45,46)$. In the present study, we observed a slight, but not statistically significant, increase in compositional stability with age.

The presence of a dominant microbial core, usually defined as a set of phylotypes shared by the majority of subjects, has been considered in several studies $(38,47)$.
The search for such a core has been a major target of the human microbiome project (48). Data on the existence of a shared, temporal core are contradictory, due to differences in definition of what is considered a common core and differences in depth of the analysis. Depth of the analysis is particularly important when studying phylogenetic groups with low abundances $(49,50)$. Two recent studies based on whole metagenome sequencing provide further evidence for the presence of a shared microbiome core in adults, with Actinobacteria, Bacteroidetes, and Verrucomicrobia populations as the most stable members $(38,51)$. In contrast, it has been reported that only a small fraction of all taxa found within a single body was constantly present over time, suggesting that a core temporal microbiome of high abundant taxa does not exist (52). In concordance, in a study describing intestinal microbiota composition in 154 adults, no single bacterial phylotype was present at an abundant frequency in fecal samples of all included subjects. Instead, the authors concluded that a core gut microbiome seems to exist at the level of metabolic functions (48). In the present study, we observed the presence of a shared microbial core in children, independent of age, dominated by species from the phylum Bacteroidetes, with the genera Bacteroides and Alistipes as the most abundant members.

The strengths of this study were the large size of the cohort, the number of fecal samples per individual, and that microbial analysis was performed using 2 different bacterial DNA-specific techniques, IS-pro and 454pyrosequencing, allowing us to cross-validate our findings. Observed species present in the shared core as measured by IS-pro were similar to core microbes established by 454pyrosequencing. Although our cohort has characteristics of heterogeneity, because healthy children aged 2-18 yr 


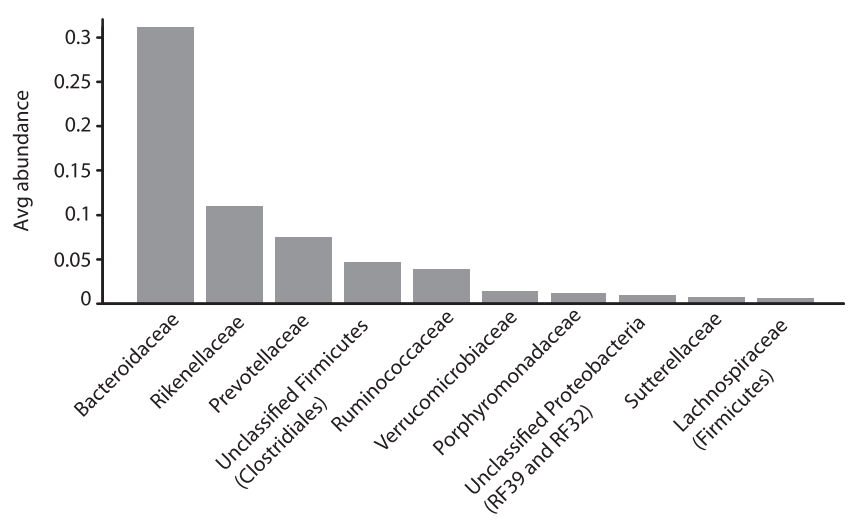

B (IS-pro)

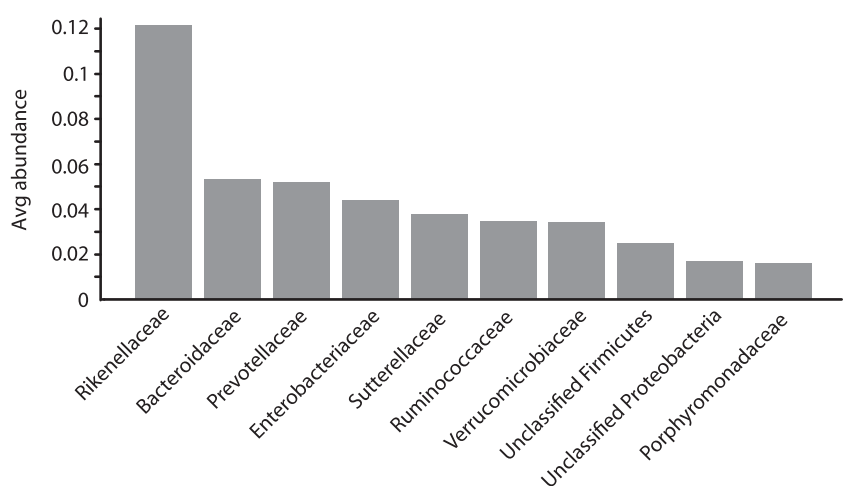

Figure 7. Bar chart representing data by 454-sequencing $(A)$ and IS-pro $(B)$ of most abundant taxa at the family level. Although observed abundances are not identical, the detected families are largely the same. A notable difference is the relatively high abundance of the family Enterobacteriaceae (mainly E. coli) in the IS-pro data.

are heterogeneous, we examined subgroups based on age and sex. Because findings in stability and composition did not differ between sex or the 3 age cohorts, we assumed the presented data to be generalizable to healthy (Dutch) children overall: the limited standard deviation in the collected specimens indicated that the number of analyzed samples was sufficient to draw universal conclusions. The present study also had limitations; the influence of environmental factors on microbiota composition, in particular later in childhood, such as antibiotic use during the first year of life, way of delivery, and neonatal feeding pattern, could not be assessed. Specifically, formula-fed children were underrepresented in this study. Additionally, food intake was not recorded daily during the study period, because detailed understanding of the influence of day-to-day changes in diet on temporal microbial dynamics would need a cohort consisting of at least hundreds of subjects; thus, the current series of children was too small for suitable statistical analysis addressing influence of diet $(53,54)$.

\section{Future perspectives}

We showed that gut microbiota composition in children is not rigid but subject to prominent fluctuations even at intervals of weeks. Presented compositional variability in health may further increase understanding of the role of the intestinal microbiota in the course of pediatric diseases linked to microbiota alterations, such as inflammatory bowel disease.

Furthermore, the observed intraindividual dynamics may have implications for future strategies assessing healthy individuals' gut microbiota signature. The currently used single-sampling strategy to analyze microbiota composition in population-wide (pediatric) studies, but also in single individuals, might not accurately reflect the entire compositional variability over time. Determination of the average composition measured at different time points could offer a more integral view. Such an approach of multiple sampling is common practice in the diagnostic workup of parasitic infections, in which a triple feces test is performed to enhance the sensitivity and specificity of the test. Regarding the observed shared microbiome core, future studies are needed to establish its precise role in health and whether disruption is linked to (increased risk for) disease and to assess environmental factors potentially influencing core composition.

In summary, in the largest study thus far on the shortterm and long-term stability of the intestinal microbiota in healthy children between 2 and 18 yr of age, we showed that stability in children is an individual characteristic varying per phylum at both short-term and long-term intervals. Stability declined rapidly for short intervals but stabilized at a level that declined further only very gradually. This was consistent throughout all age cohorts of youth.

The authors thank Prof. Dr. C. M. J. E. VandenbrouckeGrauls (Department of Medical Microbiology and Infection Control, VU University Medical Center) for the thorough review of this manuscript. The authors declare that P.H.M.S. and A.E.B. have proprietary rights on the IS-pro platform technology and are cofounders of a spinoff company developing this technique.

\section{REFERENCES}

1. Neish, A. S. (2009) Microbes in gastrointestinal health and disease. Gastroenterology 136, 65-80

2. Ley, R. E., Peterson, D. A., and Gordon, J. I. (2006) Ecological and evolutionary forces shaping microbial diversity in the human intestine. Cell 124, 837-848

3. Qin, J., Li, R., Raes, J., Arumugam, M., Burgdorf, K. S., Manichanh, C., Nielsen, T., Pons, N., Levenez, F., Yamada, T., Mende, D. R., Li, J., Xu, J., Li, S., Li, D., Cao, J., Wang, B., Liang, H., Zheng, H., Xie, Y., Tap, J., Lepage, P., Bertalan, M., Batto, J. M., Hansen, T., Le Paslier, D., Linneberg, A., Nielsen, H. B., Pelletier, E., Renault, P., Sicheritz-Ponten, T., Turner, K., Zhu, H., Yu, C., Li, S., Jian, M., Zhou, Y., Li, Y., Zhang, X., Li, S., Qin, N., Yang, H., Wang, J., Brunak, S., Doré, J., Guarner, F., Kristiansen, K., Pedersen, O., Parkhill, J., Weissenbach, J., Bork, P., Ehrlich, S. D., and Wang, J.; MetaHIT Consortium. (2010) A human gut microbial gene catalogue established by metagenomic sequencing. Nature 464, 59-65

4. Martin, R., Nauta, A. J., Ben Amor, K., Knippels, L. M., Knol, J., and Garssen, J. (2010) Early life: gut microbiota and immune development in infancy. Benef. Microbes 1, 367-382

5. Tamboli, C. P., Neut, C., Desreumaux, P., and Colombel, J. F. (2004) Dysbiosis in inflammatory bowel disease. Gut 53, 1-4

6. Ott, S. J., Musfeldt, M., Wenderoth, D. F., Hampe, J., Brant, O., Fölsch, U. R., Timmis, K. N., and Schreiber, S. (2004) Reduction in diversity of the colonic mucosa associated bacterial microflora in patients with active inflammatory bowel disease. Gut 53, 685-693 
7. Seksik, P., Rigottier-Gois, L., Gramet, G., Sutren, M., Pochart, P., Marteau, P., Jian, R., and Doré, J. (2003) Alterations of the dominant faecal bacterial groups in patients with Crohn's disease of the colon. Gut 52, 237-242

8. De la Cochetiere, M. F., Piloquet, H., des Robert, C., Darmaun, D., Galmiche, J. P., and Roze, J. C. (2004) Early intestinal bacterial colonization and necrotizing enterocolitis in premature infants: the putative role of Clostridium. Pediatr. Res. 56, 366-370

9. Ley, R. E., Turnbaugh, P. J., Klein, S., and Gordon, J. I. (2006) Microbial ecology: human gut microbes associated with obesity. Nature 444, 1022-1023

10. Clemente, J. C., Ursell, L. K., Parfrey, L. W., and Knight, R. (2012) The impact of the gut microbiota on human health: an integrative view. Cell 148, 1258-1270

11. Palmer, C., Bik, E. M., DiGiulio, D. B., Relman, D. A., and Brown, P. O. (2007) Development of the human infant intestinal microbiota. PLoS Biol. 5, e177

12. Tapiainen, T., Ylitalo, S., Eerola, E., and Uhari, M. (2006) Dynamics of gut colonization and source of intestinal flora in healthy newborn infants. APMIS 114, 812-817

13. Fallani, M., Young, D., Scott, J., Norin, E., Amarri, S., Adam, R., Aguilera, M., Khanna, S., Gil, A., Edwards, C. A., and Doré, J.; Other Members of the INFABIO Team. (2010) Intestinal microbiota of 6 week-old infants across Europe: geographic influence beyond delivery mode, breast-feeding, and antibiotics. J. Pediatr. Gastroenterol. Nutr. 51, 77-84

14. Vangay, P., Ward, T., Gerber, J. S., and Knights, D. (2015) Antibiotics, pediatric dysbiosis, and disease. Cell Host Microbe 17, 553-564

15. Arrieta, M. C., Stiemsma, L. T., Amenyogbe, N., Brown, E. M., and Finlay, B. (2014) The intestinal microbiome in early life: health and disease. Front. Immunol. 5, 427

16. Matamoros, S., Gras-Leguen, C., Le Vacon, F., Potel, G., and de La Cochetiere, M. F. (2013) Development of intestinal microbiota in infants and its impact on health. Trends Microbiol. 21, 167-173

17. Budding, A. E., Grasman, M. E., Lin, F., Bogaards, J. A., Soeltan-Kaersenhout, D. J., Vandenbroucke-Grauls, C. M., van Bodegraven, A. A., and Savelkoul, P. H. (2010) IS-pro: high-throughput molecular fingerprinting of the intestinal microbiota. FASEB J. 24, 4556-4564

18. Dethlefsen, L., Huse, S., Sogin, M. L., and Relman, D. A. (2008) The pervasive effects of an antibiotic on the human gut microbiota, as revealed by deep $16 \mathrm{~S}$ rRNA sequencing. PLoS Biol. 6, e280

19. Schloss, P. D., Westcott, S. L., Ryabin, T., Hall, J. R., Hartmann, M., Hollister, E. B., Lesniewski, R. A., Oakley, B. B., Parks, D. H., Robinson, C.J., Sahl, J. W., Stres, B., Thallinger, G. G., Van Horn, D. J., and Weber, C. F. (2009) Introducing mothur: open-source, platform-independent, community-supported software for describing and comparing microbial communities. Appl. Environ. Microbiol. 75, 7537-7541

20. Caporaso, J. G., Kuczynski, J., Stombaugh, J., Bittinger, K., Bushman, F. D., Costello, E. K., Fierer, N., Peña, A. G., Goodrich, J. K., Gordon, J. I., Huttley, G. A., Kelley, S. T., Knights, D., Koenig, J. E., Ley, R. E. Lozupone, C. A., McDonald, D., Muegge, B. D., Pirrung, M., Reeder, J., Sevinsky, J. R., Turnbaugh, P. J., Walters, W. A., Widmann, J., Yatsunenko, T., Zaneveld, J., and Knight, R. (2010) QIIME allows analysis of high-throughput community sequencing data. Nat. Methods 7, 335-336

21. McDonald, D., Price, M. N., Goodrich, J., Nawrocki, E. P., DeSantis, T. Z., Probst, A., Andersen, G. L., Knight, R., and Hugenholtz, P. (2012) An improved Greengenes taxonomy with explicit ranks for ecological and evolutionary analyses of bacteria and archaea. ISMEJ. 6, 610-618

22. Mitsue, K. (2007) Human-Computer Interaction. Interaction Platforms and Techniques. Lect. Notes Comput. Sci. 4551, 106-114

23. Shannon, P. T., Grimes, M., Kutlu, B., Bot, J. J., and Galas, D. J. (2013) RCytoscape: tools for exploratory network analysis. $B M C$ Bioinformatics 14, 217

24. Freeman, L. C. (1977) A set of measures of centrality based on betweenness. Sociometry 40, 35-41

25. Zoetendal, E. G., Akkermans, A. D., and De Vos, W. M. (1998) Temperature gradient gel electrophoresis analysis of 16S rRNA from human fecal samples reveals stable and host-specific communities of active bacteria. Appl. Environ. Microbiol. 64, 3854-3859

26. Vanhoutte, T., Huys, G., Brandt, E., and Swings, J. (2004) Temporal stability analysis of the microbiota in human feces by denaturing gradient gel electrophoresis using universal and group-specific 16S rRNA gene primers. FEMS Microbiol. Ecol. 48, 437-446

27. Scanlan, P. D., Shanahan, F., O'Mahony, C., and Marchesi, J. R. (2006) Culture-independent analyses of temporal variation of the dominant fecal microbiota and targeted bacterial subgroups in Crohn's disease. J. Clin. Microbiol. 44, 3980-3988

28. Delgado, S., Ruas-Madiedo, P., Suárez, A., and Mayo, B. (2006) Interindividual differences in microbial counts and biochemicalassociated variables in the feces of healthy Spanish adults. Dig. Dis. Sci. 51, 737-743

29. Eckburg, P. B., Bik, E. M., Bernstein, C. N., Purdom, E., Dethlefsen, L., Sargent, M., Gill, S. R., Nelson, K. E., and Relman, D. A. (2005) Diversity of the human intestinal microbial flora. Science 308, $1635-1638$

30. Durbán, A., Abellán, J. J., Jiménez-Hernández, N., Latorre, A., and Moya, A. (2012) Daily follow-up of bacterial communities in the human gut reveals stable composition and host-specific patterns of interaction. FEMS Microbiol. Ecol. 81, 427-437

31. Costello, E. K., Lauber, C. L., Hamady, M., Fierer, N., Gordon, J. I., and Knight, R. (2009) Bacterial community variation in human body habitats across space and time. Science 326, 1694-1697

32. Claesson, M. J., Cusack, S., O’Sullivan, O., Greene-Diniz, R. de Weerd, H., Flannery, E., Marchesi, J. R., Falush, D., Dinan, T., Fitzgerald, G., Stanton, C., van Sinderen, D., O'Connor, M., Harnedy, N., O'Connor, K., Henry, C., O'Mahony, D., Fitzgerald, A. P., Shanahan, F., Twomey, C., Hill, C., Ross, R. P., and O'Toole, P. W. (2011) Composition, variability, and temporal stability of the intestinal microbiota of the elderly. Proc. Natl. Acad. Sci. USA 108 (Suppl 1), 4586-4591

33. Jalanka-Tuovinen, J., Salonen, A., Nikkilä, J., Immonen, O., Kekkonen, R., Lahti, L., Palva, A., and de Vos, W. M. (2011) Intestinal microbiota in healthy adults: temporal analysis reveals individual and common core and relation to intestinal symptoms. PLoS One 6, e23035

34. Dethlefsen, L., and Relman, D. A. (2011) Incomplete recovery and individualized responses of the human distal gut microbiota to repeated antibiotic perturbation. Proc. Natl. Acad. Sci. USA 108(Suppl 1), 4554-4561

35. David, L. A., Materna, A. C., Friedman, J., Campos-Baptista, M. I., Blackburn, M. C., Perrotta, A., Erdman, S. E., and Alm, E. J. (2014) Host lifestyle affects human microbiota on daily timescales. Genome Biol. 15, R89

36. Rajilić-Stojanović, M., Heilig, H. G., Tims, S., Zoetendal, E. G., and de Vos, W. M. (2012) Long-term monitoring of the human intestinal microbiota composition [published online ahead of print October 15, 2012]. Environ. Microbiol., doi: 10.1111/14622920

37. Faith, J. J., Guruge, J. L., Charbonneau, M., Subramanian, S., Seedorf, H., Goodman, A. L., Clemente, J. C., Knight, R., Heath, A. C., Leibel, R. L., Rosenbaum, M., and Gordon, J. I. (2013) The long-term stability of the human gut microbiota. Science $\mathbf{3 4 1}$ 1237439

38. Martínez, I., Muller, C. E., and Walter, J. (2013) Long-term temporal analysis of the human fecal microbiota revealed a stable core of dominant bacterial species. PLoS One 8, e69621

39. Lin, A., Bik, E. M., Costello, E. K., Dethlefsen, L., Haque, R., Relman, D. A., and Singh, U. (2013) Distinct distal gut microbiome diversity and composition in healthy children from Bangladesh and the United States. PLoS One 8, e53838

40. Davenport, E. R., Mizrahi-Man, O., Michelini, K., Barreiro, L. B., Ober, C., and Gilad, Y. (2014) Seasonal variation in human gut microbiome composition. PLoS One 9, e90731

41. McCann, K. S. (2000) The diversity-stability debate. Nature 405, 228-233

42. Keesing, F., Holt, R. D., and Ostfeld, R. S. (2006) Effects of species diversity on disease risk. Ecol. Lett. 9, 485-498

43. Favier, C. F., Vaughan, E. E., De Vos, W. M., and Akkermans, A. D. (2002) Molecular monitoring of succession of bacterial communities in human neonates. Appl. Environ. Microbiol. 68, 219-226

44. Yatsunenko, T., Rey, F. E., Manary, M. J., Trehan, I., Dominguez-Bello, M. G., Contreras, M., Magris, M., Hidalgo, G., Baldassano, R. N., Anokhin, A. P., Heath, A. C., Warner, B., Reeder, J., Kuczynski, J., Caporaso, J. G., Lozupone, C. A., Lauber, C., Clemente, J. C., Knights, D., Knight, R., and Gordon, J. I. (2012) Human gut microbiome viewed across age and geography. Nature 486, 222-227 
45. Agans, R., Rigsbee, L., Kenche, H., Michail, S., Khamis, H. J., and Paliy, O. (2011) Distal gut microbiota of adolescent children is different from that of adults. FEMS Microbiol. Ecol. 77, 404-412

46. Balamurugan, R., Janardhan, H. P., George, S., Chittaranjan, S. P., and Ramakrishna, B. S. (2008) Bacterial succession in the colon during childhood and adolescence: molecular studies in a southern Indian village. Am. J. Clin. Nutr. 88, 1643-1647

47. Arumugam, M., Raes, J., Pelletier, E., Le Paslier, D., Yamada, T., Mende, D. R., Fernandes, G. R., Tap, J., Bruls, T., Batto, J. M., Bertalan, M., Borruel, N., Casellas, F., Fernandez, L., Gautier, L., Hansen, T., Hattori, M., Hayashi, T., Kleerebezem, M., Kurokawa, K., Leclerc, M., Levenez, F., Manichanh, C., Nielsen, H. B., Nielsen, T., Pons, N., Poulain, J., Qin, J., Sicheritz-Ponten, T., Tims, S., Torrents, D., Ugarte, E., Zoetendal, E. G., Wang, J., Guarner, F., Pedersen, O., de Vos, W. M., Brunak, S., Doré, J., Antolín, M., Artiguenave, F., Blottiere, H. M., Almeida, M., Brechot, C., Cara, C., Chervaux, C., Cultrone, A., Delorme, C., Denariaz, G., Dervyn, R., Foerstner, K. U., Friss, C., van de Guchte, M., Guedon, E., Haimet, F., Huber, W., van Hylckama-Vlieg, J., Jamet, A., Juste, C., Kaci, G., Knol, J., Lakhdari, O., Layec, S., Le Roux, K., Maguin, E., Mérieux, A., Melo Minardi, R., M'rini, C., Muller, J., Oozeer, R., Parkhill, J., Renault, P., Rescigno, M., Sanchez, N., Sunagawa, S., Torrejon, A., Turner, K., Vandemeulebrouck, G., Varela, E., Winogradsky, Y., Zeller, G., Weissenbach, J., Ehrlich, S. D., and Bork, P.; MetaHIT Consortium. (2011) Enterotypes of the human gut microbiome. Nature 473, 174-180

48. Turnbaugh, P. J., Hamady, M., Yatsunenko, T., Cantarel, B. L., Duncan, A., Ley, R. E., Sogin, M. L., Jones, W. J., Roe, B. A., Affourtit, J. P., Egholm, M., Henrissat, B., Heath, A. C., Knight, R., and Gordon,
J. I. (2009) A core gut microbiome in obese and lean twins. Nature 457, 480-484

49. Tap, J., Mondot, S., Levenez, F., Pelletier, E., Caron, C., Furet, J. P., Ugarte, E., Muñoz-Tamayo, R., Paslier, D. L., Nalin, R., Dore, J., and Leclerc, M. (2009) Towards the human intestinal microbiota phylogenetic core. Environ. Microbiol. 11, 2574-2584

50. Jalanka-Tuovinen, J., Salonen, A., Nikkilä, J., Immonen, O., Kekkonen, R., Lahti, L., Palva, A., and de Vos, W. M. (2011) Intestinal microbiota in healthy adults: temporal analysis reveals individual and common core and relation to intestinal symptoms. PLoS One 6, e23035

51. Schloissnig, S., Arumugam, M., Sunagawa, S., Mitreva, M., Tap, J., Zhu, A., Waller, A., Mende, D. R., Kultima, J. R., Martin, J., Kota, K., Sunyaev, S. R., Weinstock, G. M., and Bork, P. (2013) Genomic variation landscape of the human gut microbiome. Nature 493, 45-50

52. Caporaso, J. G., Lauber, C. L., Costello, E. K., Berg-Lyons, D., Gonzalez, A., Stombaugh, J., Knights, D., Gajer, P., Ravel, J., Fierer, N., Gordon, J. I., and Knight, R. (2011) Moving pictures of the human microbiome. Genome Biol. 12, R50

53. Salminen, S., Gibson, G. R., McCartney, A. L., and Isolauri, E. (2004) Influence of mode of delivery on gut microbiota composition in seven year old children. Gut 53, 1388-1389

54. Penders, J., Thijs, C., Vink, C., Stelma, F. F., Snijders, B., Kummeling, I., van den Brandt, P. A., and Stobberingh, E. E. (2006) Factors influencing the composition of the intestinal microbiota in early infancy. Pediatrics 118, 511-521

Received for publication June 29, 2015. Accepted for publication December 8, 2015. 